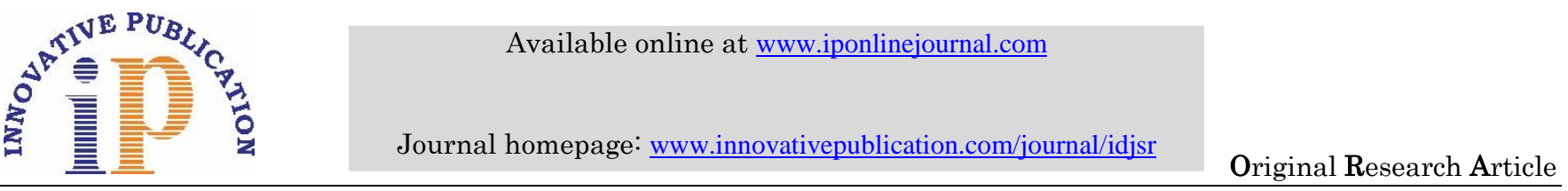

\title{
Reliability on social media for dental cosmetic remedies- A cross-sectional question based survey
}

\author{
Walaa Waleed AlHemaidi ${ }^{1}$, Shahd Hamad AlMujel ${ }^{2}$, Sarah Abdulaziz Faqih ${ }^{3}$, Njood Abdullatif Aleid ${ }^{4 *}$, Duaa \\ Abdulaziz AISharqi ${ }^{5}$
}

College of Dentistry, Riyadh Elm University, Riyadh, KSA

\begin{abstract}
Advertisements are always the method, seller and service providers to penetrate the consumer space. The internet has become an essential way of communicating, and content distributions vehicle in which social media plays a major role in the products to be published and consumed. The present online survey was conducted to know the percentage of reliability on social media for dental cosmetic remedies in Riyadh, using questionnaires.
\end{abstract}

Keywords: Dental cosmetics, Esthetics, Social media.

\section{Introduction}

It is not surprising as statistics show that more than $80 \%$ of the Middle East internet users are active on social media. With a lack of awareness among consumers, social media has been the target of individuals, and agencies exploited social media to push their content about the modern cosmetic products to the consumers, and popularize it. However, they should provide information about the side effects and increase the public awareness about the harm of the cosmetic procedures which may affect their teeth. As dentistry health professionals it is our responsibility to educate people about the harms and benefits of these cosmetic products sold online. Dentistry is one of the specialties highly affected by social media, and by doing so, it created opportunities and challenges. As professionals, we also need to exploit it to spread the highly needed awareness to the public. ${ }^{1}$

A smile could be described as a person's capability to express the amount of emotions with the appearance of the teeth and lips, and how they affect the person social life. ${ }^{2}$ Each time you smile, you benefit your health and happiness, sarcastically this smile has become costly due to the present costs of cosmetic treatments, which makes the social media play its role majorly. ${ }^{3}$ Social media has become a commonly used marketing strategy in dental product sales especially for sharing and getting information's as well as to attracts new patients. ${ }^{4}$ According to a few studies, females are more interested to use social media and Instagram is the most commonly used platform among patients who look for photographs and videos of personal artistic work of dentists for a better smile. ${ }^{3}$

Females were more interested to use social media; Instagram was the most commonly used platform among patient's while.
The aim of this study was to know the percentage of reliability on social media for dental cosmetic remedies in Riyadh city, Saudi Arabia, using specific preformed questionnaires.

\section{Materials and Methods}

After ethical review and approval obtained from the IRB (institutional review board of Riyadh Elm University, Riyadh, KSA, this cross-sectional online-based questionnaire study, with random sample technique via Survey Monkey an online survey tool was designed. Saudi, female/male, with age range from (18-65) year were included. The sample size was collected by using Raosoft sample size calculator. The questionnaire was close-ended and self-administered. The information and data from the study were entered into an electronic database (SPSS $₫$ for windows $®$ V.20).

\section{Results}

A total of 399 subjects participated in the study during 8 weeks' period of time, Table 1 shows the distribution of subjects based on gender, age and education of participants. Table 2 shows hours spent on using social media and the preferred program among participant and also the effect of social media on self-confidence in a smile. It showed that the majority of the subjects uses social media 3-5 hrs a day. Table 3 shows the perception of the smile and dental cosmology, where most of the subjects disagree with statements and $54.2 \%$ subjects answered they install orthodontics for therapeutic more than cosmetic reason. Table 4 shows the preferred color of teeth by participants, where the majority of subjects preferred light white teeth.

Tables 5 shows practice and attitude toward dental cosmology. Participants who will change their smile and will depend on dentist consultation were (79.3\%), those who

\footnotetext{
*Corresponding Author: Njood Abdullatif Aleid, College of Dentistry, Riyadh Elm University, Riyadh, KSA

Email address: njood2@hotmail.com

http://doi.org/10.18231/j.idjsr.2019.015
} 
will depend on previous experiences were (17.3\%), and only (3.4\%) will depend on a smile for a famous person. The majority of participants to some extent trust medical information about dentistry and dental cosmology derived from social media $(49.4 \%)$, and $(40.4 \%)$ of them do not trust this information derived from social media.

Table 6 shows a significant relationship between perception and practice of dental cosmology among gender groups. The majority of both gender groups disagreed to the statement (dental diastema is desirable because it looks pretty from cosmetic view). Males who disagreed to statement were significantly higher than females who disagreed to statement, $(69.4 \%)$ and $(64 \%)$ respectively, $(\mathrm{p}=0.023)$. Little deference found between males and females who agreed on the previous statement, $(8.3 \%)$ and (9.7\%) respectively, $(\mathrm{p}=0.023)$. The majority of both gender groups disagreed to the statement (Teeth diamond installation goes with smile requirements in 2018). Females who disagreed to statement were significantly higher than males who disagreed to statement, $(71.2 \%)$ and $(62 \%)$ respectively, $(\mathrm{p}=0.04)$. Also, females who agreed to the statement were more than males who agreed on the previous statement, $(8.3 \%)$ and $(5 \%)$ respectively, $(\mathrm{p}=0.04)$. Little of both males and females preferred neutral teeth color, (1.7\%) and $(4.3 \%)$, respectively, $(\mathrm{p}<0.001)$. Females who preferred white teeth color were significantly higher than males who preferred white teeth color, (44.3\%) and (23.1\%), respectively, $(\mathrm{p}<0.001)$. Males who preferred fair white teeth color were significantly higher than females who preferred fair white teeth color, (75.2\%) and (51.4\%), respectively, $(\mathrm{p}<0.001)$. The majority of both males and females think the most affecting factor on natural smile is an alignment of teeth, and males were higher than females, $(62 \%)$ and $(59 \%)$, respectively, $(\mathrm{p}=0.059)$. The majority of both females and males reported if they will change the shape of their teeth, they will depend on dentist consultation, (84.2\%) and (67\%), respectively, $(\mathrm{p}=0.001)$. Males who will change the shape of their teeth and will depend on previous experiences were significantly higher than females, $(27.3 \%)$ and $(12.9 \%)$, respectively, $(\mathrm{p}=0.001)$. Little of both females and males will depend on the smile of a famous person if they decided to change their shape of teeth, $(2.5 \%)$ and $(5.8 \%)$, respectively.

Table 7 shows the relation between perception of smile beauty and trustworthy of medical information about dentistry and dental cosmology derived from social media among age groups. Younger persons who dislike dental diastema were significantly higher than older persons, 15-25 years old $(70.8 \%), 25-35$ years old $(69.6 \%)$, and $35-45$ years old $(50 \%),(\mathrm{p}=0.007)$. Few of participants think Dental diastema is desirable because it looks pretty from cosmetic view. Those who aged from 35-45 years $(18.5 \%)$ were significantly higher than other age groups, $(\mathrm{p}=0.007)$. Those who aged 35-45 years and dislike tall interior teeth were significantly higher than other age groups who dislike tall interior teeth, and they were as follow: 35-45 years old (48.9\%), 25-35 years old 45.5\%), 15-25 years old (40.5\%), $(\mathrm{p}=0.021)$. Those who think tall anterior teeth are an indicator of beauty were as follow: $35-45$ years old (13\%), 25-35 years old $(31.3 \%), 15-25$ years old $(27.7 \%)$, $(\mathrm{p}=0.021)$. Few of participants trust medical information about dentistry and dental cosmology derived from social media and those who aged 35-45 years were significantly higher than other age groups and they were as follow: $35-45$ years old (12\%), 25-35 years old (9.8\%), 15-25 years old (9.7\%), $(\mathrm{p}=0.046)$. The majority of participants to some extent trust medical information about dentistry and dental cosmology derived from social media and they were as follow: 15-25 years old (55.4\%), 35-45 years old (50\%), 2535 years old $(38.4 \%),(\mathrm{p}=0.046)$. Those who do not trust medical information about dentistry and dental cosmology derived from social media were variable, and those who aged 25-35 years were higher than other age groups and they were as follow: $25-35$ years old $(51.8 \%), 35-45$ years old (38\%), 15-25 years old (34.9\%), ( $\mathrm{p}=0.046)$.

Table 8 shows a significant relation of participant's opinion about the best solution to aware people about new shouts and trends of dental cosmetics and how to limit the unnecessary dental cosmetic procedures among education groups. In all education groups, those who choose the best solutions is education by the dentist were significantly higher than who chosen limitation of sponsored photos in social media, (0.032). Those who choose the best solution is education by dentist among education groups were as follow: Intermediate education $(84.6 \%)$, Secondary education (61.4\%), and Bachelor education (59.1\%), $(\mathrm{p}=0.032)$.

Table 1: Shows the demographic characteristics of participants

\begin{tabular}{|c|c|c|}
\hline Character & & $\mathbf{n}(\mathbf{\%})$ \\
\hline \multirow{3}{*}{ Gender } & Female (n (\%)) & $278(69.7 \%)$ \\
\cline { 2 - 3 } & Male (n (\%)) & $121(30.3 \%)$ \\
\hline \multirow{3}{*}{ Age } & From 15 to 25 years (n (\%)) & $195(48.9 \%)$ \\
\cline { 2 - 3 } & From 25 to 35 years (n (\%)) & $112(28.1 \%)$ \\
\cline { 2 - 3 } & From 35 to 45 years (n (\%)) & $92(23.1 \%)$ \\
\hline \multirow{3}{*}{ Education } & Intermediate (n (\%)) & $013(3.3 \%)$ \\
\cline { 2 - 3 } & Secondary (n (\%)) & $83(20.8 \%)$ \\
\cline { 2 - 3 } & Bachelor (n (\%)) & $286(71.7 \%)$ \\
\cline { 2 - 3 } & Others (n (\%)) & $17(4.2 \%)$ \\
\hline
\end{tabular}


Table 2: Hours spent on using social media and preferred program

\begin{tabular}{|c|c|c|}
\hline \multicolumn{2}{|c|}{ Character } & $\mathbf{n}(\mathbf{\%})$ \\
\hline \multirow{3}{*}{ Hours spent on using social media } & 1 to 2 hours & $61(15.3 \%)$ \\
\cline { 2 - 3 } & 3 to 5 hours & $183(45.9 \%)$ \\
\cline { 2 - 3 } & M to 10 hours & $116(29.1 \%)$ \\
\cline { 2 - 3 } & More than 10 hours & $39(9.8 \%)$ \\
\hline \multirow{4}{*}{ Preferred program } & Snapchat & $140(35.1 \%)$ \\
\cline { 2 - 3 } & Twitter & $100(25.1 \%)$ \\
\cline { 2 - 3 } & Instagram & $80(20.1 \%)$ \\
\cline { 2 - 3 } & Facebook & $13(3.2 \%)$ \\
\cline { 2 - 3 } Social media affect my self-confidence in a smile & Others & $66(16.5 \%)$ \\
\cline { 2 - 3 } & To some extent & $123(30.8 \%)$ \\
\cline { 2 - 3 } & \multicolumn{2}{|c|}{$100(25.1 \%)$} \\
\hline
\end{tabular}

Table 3: Perception of a smile and dental cosmology

\begin{tabular}{|l|c|c|}
\hline \multicolumn{2}{|c|}{ Character } & $\mathbf{n}(\mathbf{\%})$ \\
\hline \multirow{3}{*}{$\begin{array}{l}\text { Dental diastema is desirable because it looks } \\
\text { pretty from the cosmetic view }\end{array}$} & Yes & $37(9.3 \%)$ \\
\cline { 2 - 3 } & May be & $82(20.6 \%)$ \\
\cline { 2 - 3 } & No & $262(65.7 \%)$ \\
\hline \multirow{3}{*}{$\begin{array}{l}\text { Teeth diamond installation goes with smile } \\
\text { requirements in } 2018\end{array}$} & I don't know & $18(4.4 \%)$ \\
\cline { 2 - 3 } & Yes & $29(7.3 \%)$ \\
\cline { 2 - 3 } & May be & $69(17.3 \%)$ \\
\hline \multirow{3}{*}{ Tall interior teeth are an indicator of beauty } & No & $273(68.4 \%)$ \\
\cline { 2 - 3 } & I don't know & $28(7 \%)$ \\
\cline { 2 - 3 } & Yes & $101(25.2 \%)$ \\
\cline { 2 - 3 } & May be & $104(26.1 \%)$ \\
\hline \multirow{3}{*}{ Most affecting factor on a neutral smile } & No & $175(43.9 \%)$ \\
\hline \multirow{3}{*}{ New dental cosmetic shouts attract me to do it } & I don't know & $19(4.8 \%)$ \\
\cline { 2 - 3 } & Size of teeth & $28(7 \%)$ \\
\cline { 2 - 3 } & Color of teeth & $232(33.1 \%)$ \\
\cline { 2 - 3 } & Alignment of teeth & $71(17.8 \%)$ \\
\hline \multirow{3}{*}{ People install orthodontics because } & yes & $79(19.8 \%)$ \\
\cline { 2 - 3 } & May be & $249(62.4 \%)$ \\
\cline { 2 - 3 } & No & $115(28.8 \%)$ \\
\cline { 2 - 3 } & Cosmetic & $68(17 \%)$ \\
\hline
\end{tabular}

Table 4: The preferred color of teeth among participants

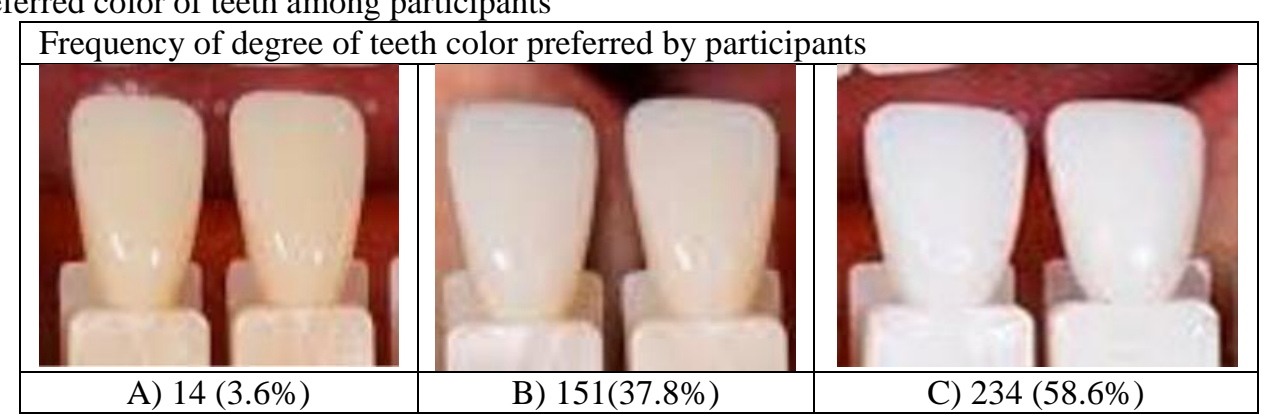

Table 5: Practice and attitude to word dental cosmology

\begin{tabular}{|c|c|}
\hline \multicolumn{1}{|c|}{ Character } & $\mathbf{n}(\%)$ \\
\hline If you decided to change your teeth shape, you will depend on & $69(17.3 \%)$ \\
\hline Previous experiences & $316(79.3 \%)$ \\
\hline Dentist consultation &
\end{tabular}




\begin{tabular}{|c|c|}
\hline \multicolumn{1}{|c|}{ Smile of a famous person } & $14(3.4 \%)$ \\
\hline $\begin{array}{l}\text { Trusting of medical information about dentistry and dental cosmology derived } \\
\text { from social media }\end{array}$ & \\
\hline Yes & $41(10.2 \%)$ \\
\hline To some extent & $197(49.4 \%)$ \\
\hline No & $161(40.4 \%)$ \\
\hline
\end{tabular}

Table 6: Significant relation between perception and practice of dental cosmology among gender groups

\begin{tabular}{|c|c|c|c|}
\hline \multirow[t]{2}{*}{ Character } & \multicolumn{2}{|c|}{ Gender } & \multirow[b]{2}{*}{ p-value } \\
\hline & $\begin{array}{c}\begin{array}{c}\text { Female } \\
\mathrm{n}=278\end{array}\end{array}$ & $\begin{array}{c}\text { Male } \\
\mathrm{n}=121\end{array}$ & \\
\hline \multicolumn{4}{|c|}{ Dental diastema is desirable because it looks pretty from the cosmetic view } \\
\hline Yes & $27(9.7 \%)$ & $10(8.3 \%)$ & \multirow{4}{*}{0.023} \\
\hline May be & $65(23.4 \%)$ & $17(14 \%)$ & \\
\hline No & $178(64 \%)$ & $84(69.4 \%)$ & \\
\hline I do not know & $8(2.9 \%)$ & $10(8.3 \%)$ & \\
\hline \multicolumn{4}{|c|}{ Teeth diamond installation goes with smile requirements in 2018} \\
\hline Yes & $23(8.3 \%)$ & $6(5 \%)$ & \multirow{4}{*}{0.04} \\
\hline May be & $42(15.1 \%)$ & $27(22.3 \%)$ & \\
\hline No & $198(71.2 \%)$ & $75(62 \%)$ & \\
\hline I do not know & $15(5.4 \%)$ & $13(10.7 \%)$ & \\
\hline \multicolumn{4}{|c|}{ The preferred color of teeth among participants } \\
\hline Neutral teeth color (Option-1) & $12(4.3 \%)$ & $2(1.7 \%)$ & \multirow{3}{*}{$<0.001$} \\
\hline White teeth (Option-2) & $123(44.3 \%)$ & $28(23.1 \%)$ & \\
\hline Fair white teeth color (Option-3) & $143(51.4 \%)$ & $91(75.2 \%)$ & \\
\hline \multicolumn{4}{|l|}{ Most affecting factor on a neutral smile } \\
\hline Alignment of teeth & $164(59 \%)$ & $75(62 \%)$ & \multirow{3}{*}{0.059} \\
\hline Size of teeth & $20(7.1 \%)$ & $8(6.6 \%)$ & \\
\hline Color of teeth & $94(33.9 \%)$ & $38(31.4 \%)$ & \\
\hline \multicolumn{4}{|c|}{ If you decided to change your teeth shape, you will depend on } \\
\hline Dentist consultation & $234(84.2 \%)$ & $81(67 \%)$ & \multirow{3}{*}{0.001} \\
\hline Previous experiences & $36(12.9 \%)$ & $33(27.3 \%)$ & \\
\hline Smile of a famous person & $7(2.5 \%)$ & $7(5.8 \%)$ & \\
\hline
\end{tabular}

Table 7: Relation between the perception of smile beauty and trustworthy of medical information about dentistry and dental cosmology derived from social media among age groups

\begin{tabular}{|c|c|c|c|c|}
\hline \multirow{2}{*}{ Character } & \multicolumn{3}{|c|}{ Age (by years) } & \multirow[b]{2}{*}{ p-value } \\
\hline & $\begin{array}{c}15-25 y \\
n=195\end{array}$ & $\begin{array}{c}25-35 y \\
n=112\end{array}$ & $\begin{array}{c}35-45 y \\
n=92\end{array}$ & \\
\hline \multicolumn{5}{|c|}{ Dental diastema is desirable because it looks pretty from the cosmetic view } \\
\hline Yes & $13(6.7 \%)$ & $7(6.3 \%)$ & $17(18.5 \%)$ & \multirow{4}{*}{0.007} \\
\hline May be & $35(17.9 \%)$ & $23(20.5 \%)$ & $24(26.1 \%)$ & \\
\hline No & $138(70.8 \%)$ & $78(69.6 \%)$ & $46(50 \%)$ & \\
\hline I do not know & $9(4.6 \%)$ & $4(3.6 \%)$ & $5(5.4 \%)$ & \\
\hline \multicolumn{5}{|c|}{ Tall interior teeth are an indicator of beauty } \\
\hline Yes & $54(27.7 \%)$ & $35(31.3 \%)$ & $12(13 \%)$ & \multirow{4}{*}{0.021} \\
\hline May be & $55(28.2 \%)$ & $22(19.6 \%)$ & $27(29.3 \%)$ & \\
\hline No & $79(40.5 \%)$ & $51(45.5 \%)$ & $45(48.9 \%)$ & \\
\hline I do not know & $7(6.3 \%)$ & $4(3.6 \%)$ & $8(8.7 \%)$ & \\
\hline \multicolumn{5}{|c|}{ Trusting of medical information about dentistry and dental cosmology derived from social media } \\
\hline Yes & $19(9.7 \%)$ & $11(9.8 \%)$ & $11(12 \%)$ & \multirow{3}{*}{0.046} \\
\hline To some extent & $108(55.4 \%)$ & $43(38.4 \%)$ & $46(50 \%)$ & \\
\hline No & $68(34.9 \%)$ & $58(51.8 \%)$ & $35(38 \%)$ & \\
\hline
\end{tabular}


Table 8: Significant relation of participant's opinion about the best solution to aware people about new shouts and trends of dental cosmetics and how to limit the unnecessary dental cosmetic procedures among education groups

\begin{tabular}{|c|c|c|c|c|}
\hline \multirow{2}{*}{ Character } & \multicolumn{3}{|c|}{ Education } & \multirow[b]{2}{*}{ p-value } \\
\hline & $\begin{array}{c}\text { intermediate } \\
\mathrm{n}=13\end{array}$ & $\begin{array}{c}\text { secondary } \\
\mathrm{n}=83\end{array}$ & $\begin{array}{c}\text { bachelor } \\
\mathrm{n}=286\end{array}$ & \\
\hline \multicolumn{5}{|c|}{$\begin{array}{l}\text { The best solution to aware people about new shouts and trends of dental cosmetics and how to limit the unnecessary } \\
\text { dental cosmetic procedures }\end{array}$} \\
\hline Limitation of sponsored photos on social media & $2(15.4 \%)$ & $32(38.6 \%)$ & $117(40.9 \%)$ & \multirow[b]{2}{*}{0.032} \\
\hline Education by the doctor & $11(84.6 \%)$ & $51(61.4 \%)$ & $169(59.1 \%)$ & \\
\hline
\end{tabular}

\section{Discussion}

The dental profession has observed an increasing interest in cosmetic restorations in the last 10 years. This is reflected by ongoing efforts to develop new materials and clinical methods. ${ }^{1,2}$ To the best of our knowledge, there are no much researches done to test the association between the effects of social media on perception and attitude of a smile. In the current study, we found that $(9.3 \%)$ of participants prefer dental diastema because it looks pretty. A study was done internationally in 1998 to test participants perception toward dental appearance, and they found almost all of the participants did not prefer dental diastema. ${ }^{5}$ Also, in our study, we tested the preferred teeth color among participants, we found that the majority of participants prefer light teeth color $(58.6 \%),(37.8 \%)$ of them prefer medium white teeth, and only a few of them prefer dark teeth $(3.6 \%)$. The previously mentioned study measured their participants preferred color of teeth, and their results were different to our findings, as the majority of their participants preferred medium white teeth color $(62 \%)$, the majority of remaining preferred light teeth color $(32 \%)$ and only $(6 \%)$ preferred dark white teeth color. ${ }^{3,5}$ In the current study, we tested participants perception toward dental esthetics (orthodontics), we reported that more than half of participants think dental esthetics (orthodontics) installed for therapeutic reasons $(54.2 \%)$ as it will improve function of teeth and not only for merely cosmetic and for appearance reasons, those who think these procedures installed for appearance and cosmetic reasons were (28.8\%), while few of them think it is installed for both therapeutic and cosmetic reasons $(17 \%)$. The previously mentioned study reported nearly three fourths $(75 \%)$ of participants said good teeth function is more important than teeth appearance, also when they asked separately about the importance of teeth appearance, (95\%) of them said it is either Important or Very important, and their response to the function of teeth were (99\%) of them said it is either Important or Very important. ${ }^{5}$ To conclude there was no significant association between social media use and perception of smile and dental cosmology and practice. There was variable perception regarding the measures of a pretty smile, as these measures are dependent and may change from culture to culture, and from age group to age group. Also, we need to pay efforts to await people to decrease the time spent using social media, and aware them about the side effects of the excessive use of social media on the psychological, medical and social health. ${ }^{6}$

\section{Conclusion}

Within the limitation of the current study, we concluded that we need to develop campaigns and pay more efforts to educate people about the indications and contraindications of dental procedures and products advertised online, more attention is needed to psychological and social circumstances that could affect our perception toward dental knowledge and procedures.

\section{Conflict of Interest}

None.

\section{References}

1. Koirala S. Smile Design Wheel ${ }^{\mathrm{TM}}$ : A practical approach to smile design. Cosmet Dent 2009;3(3):24-8.

2. Afroz S, Rathi S, Rajput G, Rahman SA. Dental esthetics and its impact on psycho-social well-being and dental self confidence: A campus based survey of North Indian University students. J Indian Prosthod Soc 2013;13(4):455-60.

3. Nancy Ajwa SA, Kuwail A, Osaif EA. The impact of using social media networks on dental treatment marketing in Saudi Arabia: The practitioners and patient's perspectives. J Oral Health Dent Sci 2019;2(3):1-10.

4. Arnett MR, Loewen J, Romito L. Use of social media by dental educators. J Dent Educ 2013;77(11):1402-12.

5. Carlsson GE, Wagner I-V, Ödman P, An international comparative multicenter study of assessment of dental appearance using computer-aided image manipulation. Int $J$ Prosthod 1998;11(3).

6. Kolmes K. Social media in the future of professional psychology. Prof Psychol: Res Pract 2012;43(6):606.

How to cite this article: AlHemaidi WW, AlMujel SH, Faqih SA, Aleid NA, AlSharqi DA. Reliability on social media for dental cosmetic remedies- A cross-sectional question based survey. Int Dent J Student Res 2019;7(3):60-4. 